\title{
Experiencia de la enseñanza a distancia a través del uso de TIC en la formación de diseñadores urbanos y del paisaje para la elaboración de programas de ordenamiento territorial
}

\author{
Experience of distance education through the use of ICT \\ in the training of urban and landscape designers \\ for the development of territorial management programs
}

\author{
Ana Ma Delgadillo Silva ${ }^{1}$ \\ Martha Yolanda Pérez Barragán ${ }^{2}$ \\ Universidad Autónoma de San Luis Potosí
}

Recibido: 02.09.2021

Aceptado: 30.10 .2021

\section{Resumen}

La Universidad Autónoma de San Luis Potosí, México, dentro de su Plan Institucional de Desarrollo 2013-2023, ha incorporado espacios y ambientes de formación que responden a las dimensiones del Modelo Universitario de Formación Integral y esto ha permitido acercar a los docentes y alumnos estrechamente a las funciones y aplicaciones a las diversas Tecnologías de la Información y Comunicación (TIC) que han sido útiles en los procesos de enseñanzaaprendizaje, contenidos que se gestionan a través de recursos y espacios virtuales accesibles principalmente a través de Internet. La experiencia dentro de la Facultad del Hábitat en la

\footnotetext{
${ }^{1}$ anadelgadillo@fh.uaslp.mx

https://orcid.org/0000-0003-2200-2069

2 marthaperez@uaslp.mx 
Carrera de Diseño Urbano y del Paisaje con apoyo de los profesores investigadores del Observatorio urbano local que tiene sede en esta Facultad, además de realizar la instrucción a distancia desarrollando comunidades virtuales y aunado a las nuevas disposiciones de Políticas Nacionales en relación con el Ordenamiento Territorial es que en el Plan de estudios 2013 de la formación de Diseñadores Urbanos y del Paisaje, ofrece la materia de Ordenamiento Territorial en el área de profesionalización para desarrollar competencias profesionales en los alumnos en cuanto a la preparación de los procesos metodológicos para la elaboración de programas de ordenamiento territorial y que se ajustan a los que exige la ley de OT, con la finalidad de construir cuadros de especialistas tanto en lo que respecta al conocimiento del territorio y sus diversas variables como en el uso de las nuevas tecnologías en sistemas de información geográfica $(\mathrm{SIG})$, principalmente obedece a la relevancia de codificar, y monitorear ensamblajes de datos y métodos provenientes de diferentes áreas del conocimiento por medio de las herramientas tecnológicas de información de los que respecta al territorio como estructura geográfica-espacial, marco que implica aplicar las normas vigentes para evaluar y hacer gestión territorial. En el presente trabajo se muestra algunas de las actividades realizadas del proceso enseñanza aprendizaje a través de las TIC y los resultados obtenidos.

Palabras Clave: Tecnologías de la Información y Comunicación, Comunidades Virtuales de Aprendizaje, SIG para el Ordenamiento Territorial.

\section{Abstract}

The Autonomous University of San Luis Potosí, Mexico, within its Institutional Development Plan 2013-2023, has incorporated training spaces and environments that respond to the dimensions of the University Model of Comprehensive Training and this has allowed to bring teachers and students closely to the functions and applications to the various Information and Communication Technologies (ICT) that have been useful in the teaching-learning processes, contents that are managed through resources and virtual spaces accessible mainly through the Internet. The experience within the Faculty of Habitat in the Career of Urban and Landscape 
Design with the support of the research professors of the local urban Observatory that is based in this Faculty, in addition to carrying out distance instruction developing virtual communities and added to the new provisions of National Policies in relation to Territorial Planning is that in the 2013 Curriculum for the training of Urban and Landscape Designers, it offers the subject of Territorial Planning to develop professional competencies in students regarding the preparation of methodological processes for the development of land use planning programs that conform to those required by the OT law, in order to build cadres of specialists both in terms of knowledge of the territory and its various variables and in the use of new technologies in geographic information systems (GIS), main mind obeys the relevance of coding, and monitoring data assemblages and methods from different areas of knowledge by means of information technology tools regarding the territory as a geographical-spatial structure, a framework that implies applying the current norms to evaluate and do territorial management. This work shows some of the activities carried out in the teaching-learning process through ICT and the results obtained.

Keywords: Information and Communication Technologies, Virtual Learning Communities, GIS for Territorial Planning.

\section{Introducción}

A lo largo de la historia la educación ha sufrido cambios que desafían los procesos de enseñanza aprendizaje que llevan a la búsqueda de alternativas metodológicas para la calidad educativa, por lo que las entidades educativas buscan capacitar al profesor para generarle competencias que permitan el adecuado desempeño de dicho proceso. Uno de los fines principales de la Educación Superior es gestionar prácticas reflexivas para el aprendizaje de sus alumnos, en virtud de que el hombre es conformado por las experiencias que vive diariamente en los diferentes ámbitos dentro de los cuales se desarrolla. Existe la necesidad de ser perceptivos a las transformaciones que hay en la humanidad y en especial en el ámbito educativo. En la actualidad las Tecnologías de la información y la Comunicación (TIC) son un mecanismo clave en el avance de la sociedad del conocimiento, de gran impacto que 
alcanza nuevas magnitudes, y su influencia es de tal forma que no sólo afecta a la velocidad con la que nos llega los información, sino también la cantidad que se pone a disposición, o la precisión con la que se logra realizar determinadas actividades; sino también a la forma como se llega a procesar la información, la manera en la cual se aprende, las diversas formas en que se enseña, y las opciones que se tiene para comunicar. La incorporación de las TIC a los procesos de enseñanza superior requiere de transformaciones, pues, de nada sirve introducir nuevas tecnologías si no se producen otros cambios en el sistema de enseñanza. Cualquier proceso de incorporación en este ámbito, debe ser analizado y estudiado como una innovación, ya que la oferta educativa presenta cambios y transformaciones en todos los elementos del proceso didáctico precisando abreviar su trayecto con el fin de satisfacer los requerimientos de los estudiantes del siglo XXI, enfocados cada vez más hacia el uso de la tecnología (Salinas y Salinas, 2013). Efectuar esta demanda implica crear espacios para que el docente reflexione en las nuevas formas de enseñanza orientadas a los alumnos digitales.

Para tal caso la Universidad Autónoma de San Luis Potosí a través de la Secretaría Académica desde hace ya más de 10 años promueve la formación de los profesores; en la incorporación de las nuevas tecnologías de información y comunicación (TIC). Además, formula propuestas y marcos de referencia que orienten los procesos de innovación en las entidades académicas. Ofrece a los profesores el portal eVirtual de la UASLP que desde el 2009 tiene como objetivo ofrecer espacios virtuales de comunicación e intercambio de información y está a disposición de profesores, estudiantes, trabajadores y autoridades de la institución. Para ello adopta esquemas que permiten optimizar la infraestructura en tecnologías de la información y comunicación, mediante la disposición de una red convergente, es decir, multi servicios, basada en arquitecturas informáticas orientadas a servicios que permiten estar a la vanguardia.

La tecnología educativa en la UASLP incorpora como estrategia la incorporación de las TIC en los procesos educativos mediante actividades de fomento de la capacidad, asesoramiento técnico, publicaciones, cursos para los profesores y no se limita a lo que la misma entidad ofrece, sino a todo el conjunto de herramientas que son accesibles vía internet y que el profesor decide utilizar. Actualmente, ante la situación pandémica del 2020 por el COVID -19 se 
consideró la emergencia para brindar acceso a la educación de los alumnos Universitarios y reconsiderar la propuesta de implementación y evaluación de los cursos y materias de las carreras para buscar orientar acciones para la mejora del proceso educativo sin menoscabo de la calidad generados a partir de la migración total a la modalidad virtual. Es con este propósito que al migrar a la enseñanza aprendizaje de presencial a distancia se ofreció a la comunidad Universitaria los aspectos básicos para implementar los cursos a través de la Plataforma TEAMS de Windows incorporado al correo institucional de alumnos y profesores con el propósito de hacer uso de las plataformas de la UASLP y adaptar las unidades de aprendizaje al sistema en línea, por lo cual se planificaron y diseñaron las actividades/ evidencias y se crearon los recursos didácticos adaptando los materiales con los que ya se contaba en clases presenciales. Sin embargo los criterios para la selección y evaluación de las herramientas tecnológicas pensadas para lograr la enseñanza aprendizaje para cada curso debe superar los supuestos teórico conceptuales y el saber específico de área del conocimiento de cada materia; debemos saber que lo visible como comenta Valderrama (2016) es la técnica o el método a través de la estrategia didáctica elegida para tal fin sin embargo al construir los contenidos para la enseñanza a distancia es importante tomar en cuenta que se va a enseñar, a quien se va a enseñar y como se aprende y no pensar precisamente solo en las herramientas.

La experiencia dentro de la Facultad del Hábitat de la UASLP en la Carrera de Diseño Urbano y del Paisaje con apoyo de los profesores investigadores del Observatorio urbano local que tiene sede en esta Facultad, además de realizar la instrucción a distancia desarrollando comunidades virtuales y aunado a las nuevas disposiciones de Políticas Nacionales en relación con el Ordenamiento Territorial es que en el Plan de estudios 2013 de la formación de Diseñadores Urbanos y del Paisaje, ofrece la materia de Ordenamiento Territorial en el área de formación profesional, para desarrollar competencias profesionales en los alumnos en cuanto a la preparación de los procesos metodológicos para la elaboración de programas de ordenamiento territorial y que se ajusten a los que exige la ley de OT nacional y local, con la finalidad de construir cuadros de especialistas tanto en lo que respecta al conocimiento del territorio y sus diversas variables como en el uso de las nuevas tecnologías en sistemas de información geográfica (SIG), principalmente obedeciendo a la relevancia de codificar, y 
monitorear ensamblajes de datos y métodos provenientes de diferentes áreas del conocimiento por medio de las herramientas tecnológicas de información de los que respecta al territorio como estructura geográfica-espacial, marco que implica aplicar las normas vigentes para evaluar y hacer gestión territorial.

La estrategia de enseñanza aprendizaje digital para crear la comunidad virtual obedeció entonces a construir primero los contenidos para que estos pudiesen ser enseñados y aprendidos a distancia a través de las tecnologías de la comunicación por lo que incluir diversas herramientas que se incorporaron, la Universidad puso a disposición la Plataforma Micosoft Teams, como el espacio principal de comunicación y colaboración combinado con el chat persistente en el lugar de trabajo, reuniones de video, almacenamiento de archivos e integración de aplicaciones, por las características del curso se incluyó en la enseñanza aprendizaje el uso de la plataforma web del Observatorio Urbano Local de San Luis Potosí y los recursos tecnológicos del laboratorio de imagen satelital que tienen sede en la FH y que fungió como enlace para la obtención de datos estadísticos de la región, ya que está vinculado directamente con la Plataforma digital del INEGI (Sistema nacional de información estadística y geografía) y a su plataforma Mapa Digital de México herramienta informática que permite la construcción, consulta, interpretación y análisis de la información geográfica y geoestadística georreferenciada. Así mismo ante la imposibilidad de asistir presencialmente al laboratorio de imagen satelital, la Facultad del Hábitat a través de las licencias que tiene con Arc Gis, abrió la posibilidad para que profesores y alumnos accediéramos a la plataforma Arc Gis on line a través del correo institucional con el objetivo de trabajar los datos creando mapas temáticos para el análisis territorial. 
Imagen 1. Plataforma Microsoft Teams, como el espacio principal de comunicación y colaboración del Curso Ordenamiento Territorial, Diseño urbano y del paisaje, Facultad del Hábitat, UASLP.

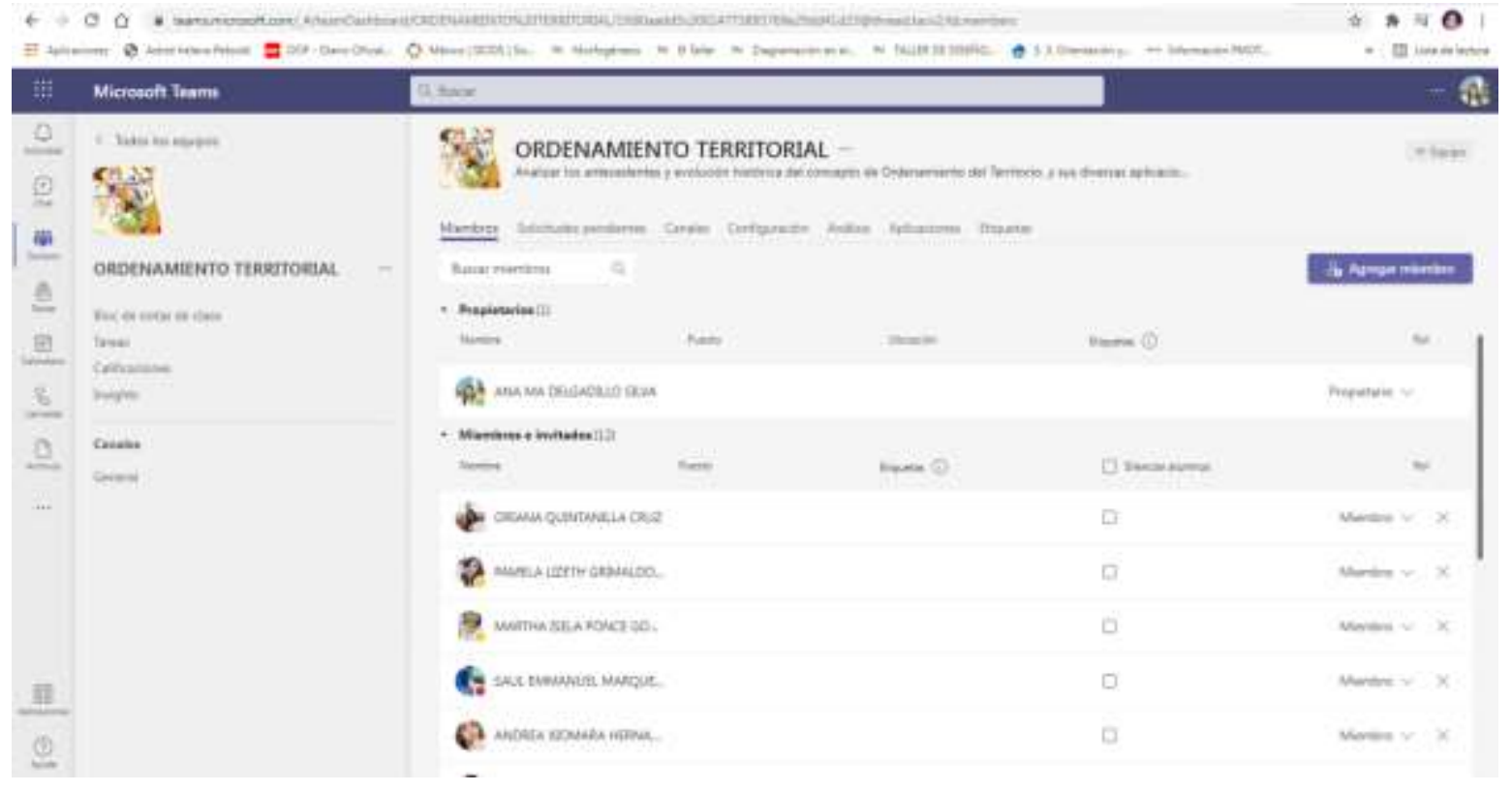

Imagen 2. Plataformas OUL, INEGI y Arc Gis on line de la Facultad del Hábitat ${ }^{3}$

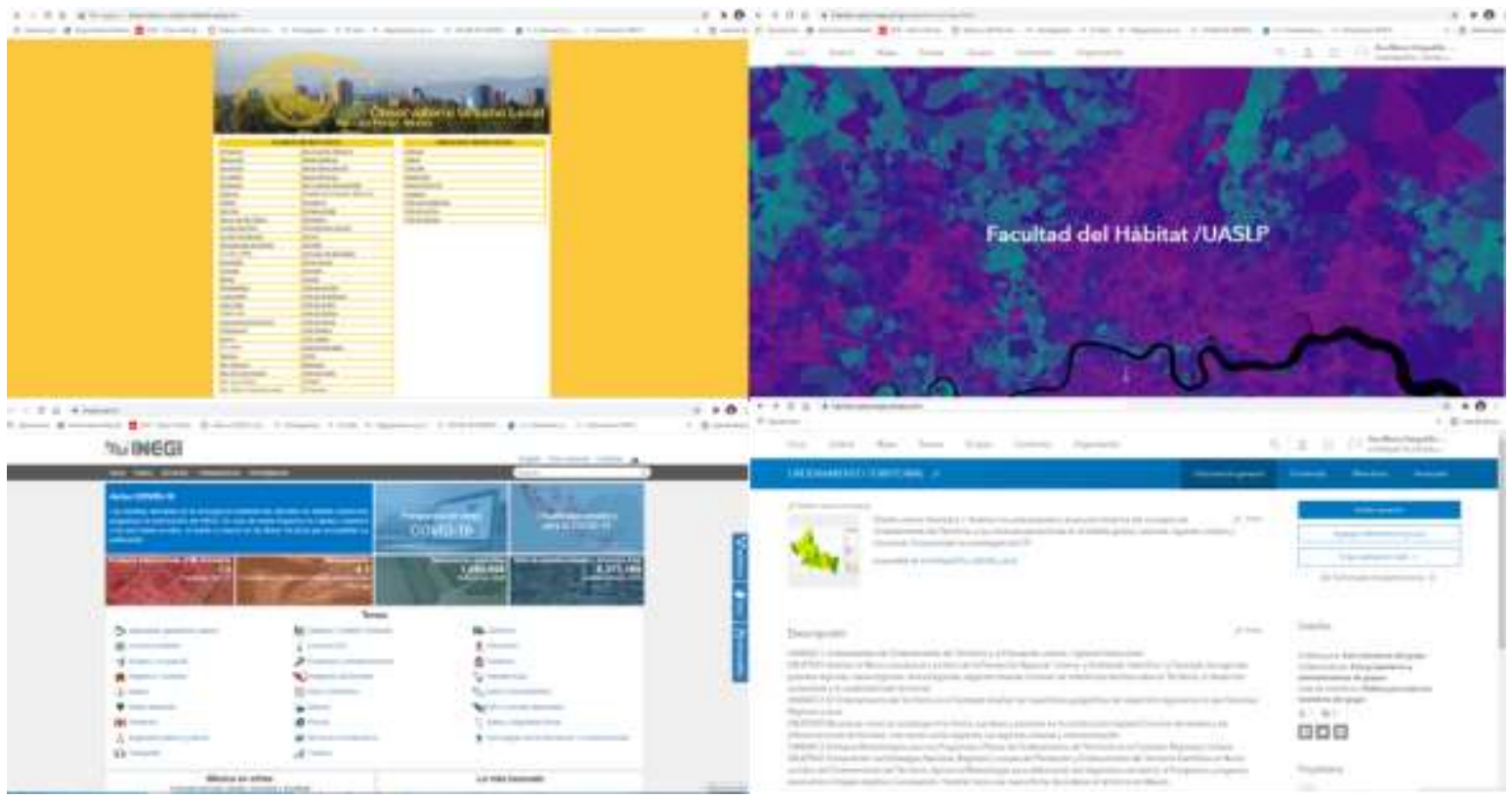

${ }^{3}$ utilizadas en el curso de Ordenamiento territorial como enlace de obtención de datos y que figuran como un soporte técnico y como herramienta que favorece el diseño de materiales y cumplir con los objetivos de aprendizaje y construir los productos finales. 
Imagen 3. Resultado de trabajo ${ }^{4}$.

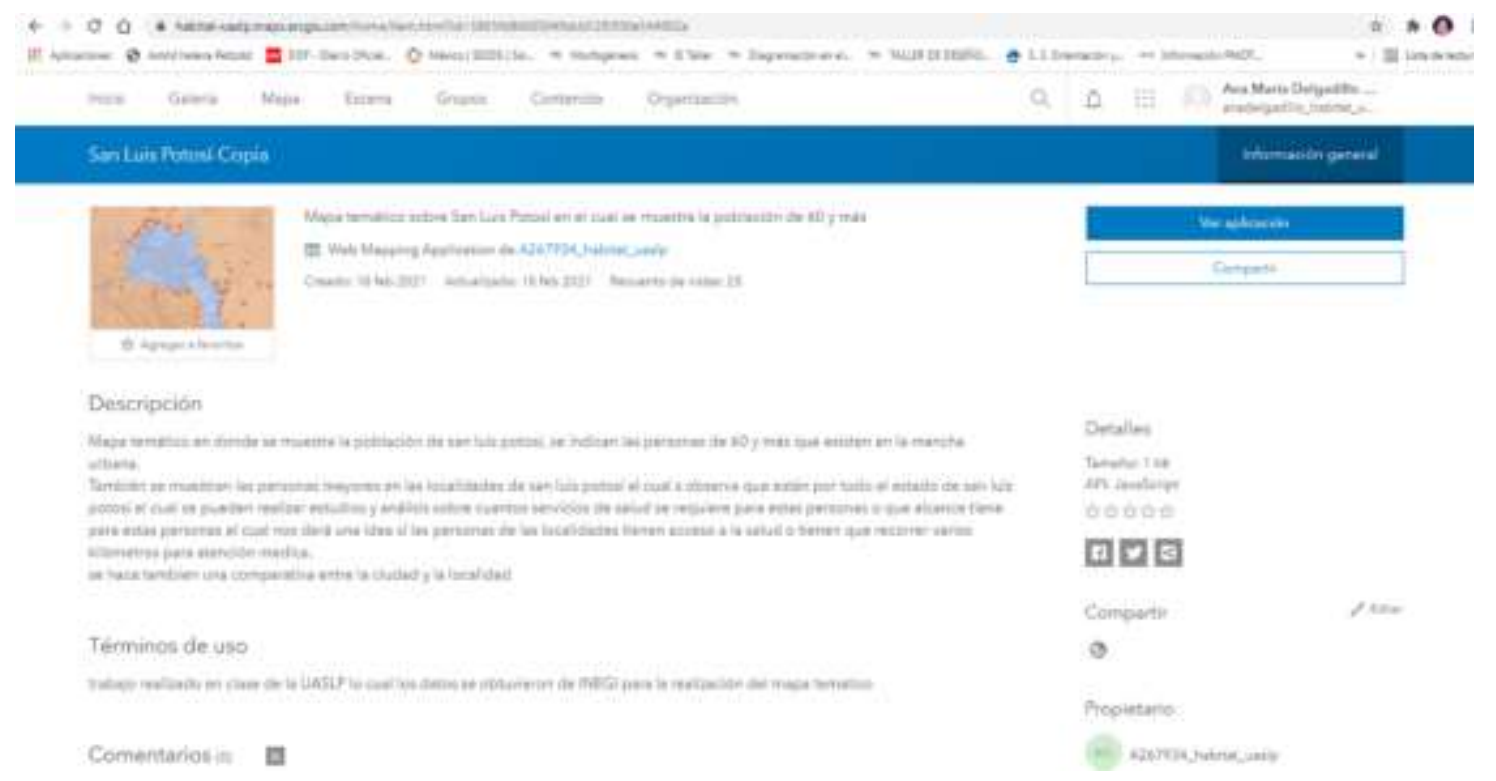

\section{Desarrollo}

Las autoras como investigadoras de tiempo completo de la Universidad Autónoma de San Luis Potosí adscritas a la Facultad del Hábitat e integrantes del Cuerpo académico Habitabilidad y sustentabilidad del territorio y en la Coordinación del Observatorio Urbano Local, generamos conocimiento a partir de la vinculación con los proyectos de orden territorial del Estado de San Luis Potosí y sus municipios, tarea que se presenta como oportunidad como docentes para la formación de profesionales de Diseño Urbano y del Paisaje a través de su involucramiento en proyectos reales, por lo que en el presente trabajo se muestra algunas de las actividades realizadas del proceso enseñanza aprendizaje del Ordenamiento territorial a través de las TIC y los resultados obtenidos.

Para responder a estos desafíos, la Universidad Autónoma de San Luis Potosí, a la que pertenece la Facultad del Hábitat, revisó sus referentes actuales y promueve experiencias innovadoras en procesos de enseñanza-aprendizaje apoyados en las TIC. En este terreno la

\footnotetext{
${ }^{4}$ Trabajo resultado de la interacción de codificar, y monitorear ensamblajes de datos y métodos provenientes de diferentes áreas del conocimiento por medio de las herramientas tecnológicas de información de los que respecta al territorio como estructura geográfica-espacial 
Universidad se enfrentó como otras a dificultades asociadas a la capacidad de flexibilización de sus estructuras y que aplicado al tema que nos ocupa, las palabras clave para la innovación en la formación que ponen en relación las tecnologías de la información y la comunicación y los nuevos enfoques respecto al aprendizaje son, de acuerdo con Fullan y Smith (1999): significado, coherencia, conectividad, sinergia, alineamiento y capacidad para la mejora continua que contrasta con los modelos lineales de cambio (Salinas J. 2004).

Así, la innovación, si bien está próxima a la práctica, está relacionada con todo el proceso, con perspectivas de globalidad, e implica cambios en el currículo, por lo que la Facultad del Hábitat está en procesos continuos de revisión y actualización curricular en las formas de ver y pensar las disciplinas, en las estrategias desplegadas, en la forma de organizar y vincular cada disciplina con otra, en el modelo hábitat de la interdisciplina. La aplicación de las TIC en acciones de formación bajo la concepción de enseñanza flexible ( presencial, a distancia o mixta ) abrió varias fases de cambio y renovación que consideraron cambios en la idea de cómo funciona en el aula, la definición de los procesos didácticos, en la identidad del docente, etc., pero también abrió la puerta a la necesidad de cambios en los recursos básicos tales como contenidos (materiales, etc.), infraestructuras (acceso a redes, etc.), uso abierto de estos recursos (manipulables por el profesor, por el alumno...) así como la capacitación constante en las prácticas de los profesores y de los alumnos en el proceso de enseñanza aprendizaje.

Para ello se ha aprovechado la variedad de tecnologías de la comunicación y lograr que proporcione la flexibilidad necesaria para cubrir necesidades individuales y sociales, para lograr entornos de aprendizaje efectivos y conseguir la interacción profesor-alumno adoptando estrategias didácticas adecuadas. La planeación didáctica dirigida por la secretaria académica de la FH, para cada carrera y cada materia pretende lograr los objetivos de aprendizaje por los cuales asegurar el logro de objetivos por parte del alumno y elegir la estrategia didáctica por parte del profesor a través de las TIC y decidir el tipo de combinación de métodos, medios y técnicas que apoyen al alumno a alcanzar la meta deseada. Apoyados por la propia Institución Universitaria con todos estos recursos, en nuestro caso pretende atender la complejidad de la práctica educativa en lo que respecta a la formación de 
profesionales del Diseño Urbano y del Paisaje en lo que concierne al Ordenamiento territorial permitiendo así la combinación de varias soluciones que pueda también cumplir con el Modelo educativo Institucional acercando al profesor como transmisor del conocimiento a los diversos recursos tanto tecnológicos como de infraestructura física para al impartición de los cursos a distancia centrados en el alumno, considerando a éste como la parte activa de procesos de enseñanza aprendizaje.

Si centramos los esfuerzos de las técnicas didácticas elegidas a través de la red de internet y de los dispositivos electrónicos (computadora y celular) habrá que entonces adecuar los contenidos y los recursos didácticos que se utilizan en los cursos presenciales de acuerdo a las posibilidades de la tecnología disponible y las posibilidades comunicativas y de interacción.

Salinas (2008) comenta que la combinación de técnicas resulta de mayor riqueza de cara a lograr un entorno enseñanza aprendizaje efectivo, y que la combinación de características de los usuarios, organización, tecnología determinan cuáles serán las posibles estrategias a seguir dependiendo de los objetivos del curso. Por lo que señala que Pérez (2004) propone y agrupa las técnicas didácticas en torno a la individualización de los procesos de enseñanza basado en el trabajo autónomo del alumno a partir de la propuesta de materiales y trabajo a realizar presentados en la planeación didáctica, por lo que entre profesor y el alumno hay una interacción de orientación, seguimiento y control individual.

Las exposiciones y participaciones grupales, comprende las técnicas de trabajo grupal a partir de las exposiciones del profesor o de invitados expositores ya sea disertación o por medio de video o diapositivas y lecturas lo que implica acceso a material e información por parte de los alumnos para interactuar grupalmente, técnica que abarca la participación activa en la construcción del conocimiento. 


\subsection{Creación de la comunidad virtual educativa para el ordenamiento territorial}

Crear la comunidad virtual de aprendizaje de exige compartir intereses comunes comunicándose a través de las diferentes herramientas disponibles de comunicación que pueden ofrecer reuniones sincrónicas y asincrónicas, permitiendo una innovadora visión de la formación que abre puertas, generando experiencias e interacciones de aprendizaje dentro de la Universidad, complementando el proceso de enseñanza aprendizaje. En la definición de Rheignold $^{5}$, en la comunidad virtual de aprendizaje se encuentran tres elementos: la interactividad, el tiempo, lo afectivo como las condiciones para que se dé efectiva a la comunidad. Sin embargo, para Powers ${ }^{6}$ la comunidad virtual es el espacio electrónico donde un grupo de personas se reúnen para intercambiar ideas por lo que denota una extensión de la vida habitual en conjunto con dispositivos de computo conformando un entorno de relación social y en este caso educativo también, por lo que es un entorno de usabilidad y de sociabilidad (Guevara, B, Guzmán J.C, Zambrano N. 2007).

\subsection{Plataformas y recursos}

Fig. 1. Componentes Claves del diseño de Comunidad Virtual para el curso Ordenamiento territorial en la Facultad del Hábitat. Adaptado de Preece (2003). En Guevara (2007).

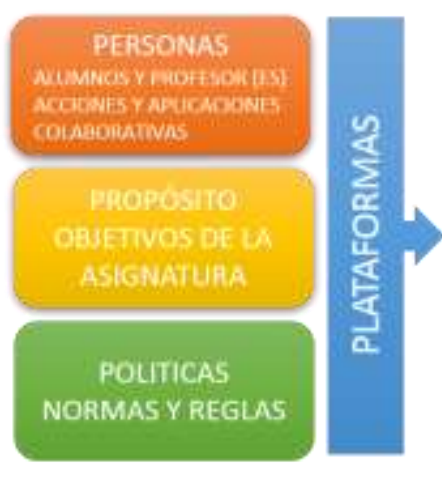

Sociabilidad

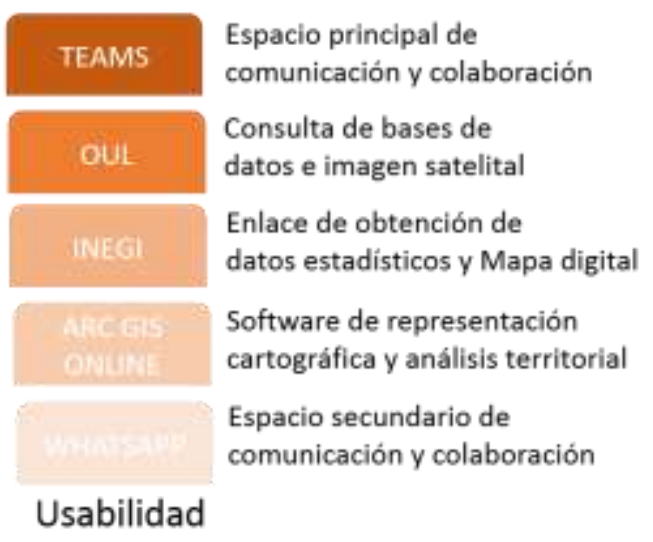

Usabilidad

\footnotetext{
${ }^{5}$ Rheignold H. 1993

${ }^{6}$ Powers, M. 1997

Volumen 2. Número 2. Julio - Diciembre 2022 ISSN: 2745-0341 (En línea)
} 
Para la materia de Ordenamiento en línea implicó adaptar los contenidos de la Unidades de aprendizaje en el espacio principal de comunicación a través de la plataforma Microsoft Teams. Los criterios que se tomaron en cuenta al seleccionar las herramientas fueron a través de los siguientes aspectos:

Los usuarios deben poder navegar e interactuar con los contenidos presentados, las herramientas seleccionadas se adaptan a los propósitos de aprendizaje dentro e la comunidad virtual a través de la red, los contenidos de enseñanza se presentaron de forma dinámica, amigable e interactiva a través de videos cortos, presentaciones en power point, y foros de debate sincrónicos y asincrónicos, test de repaso interactivo. Además, a través del uso de diversas plataformas acordes al área del conocimiento se ofrece el acceso a documentos, investigaciones, tutoriales, conferencias así como información hipertextual con estructura no lineal como lo es con la Plataforma web del Observatorio Local y su Laboratorio Satelital así como con los contenidos de la Plataforma del INEGI, apoyados con la Red social whatsap como vínculo de comunicación donde se puede también además de mensajes enviar información y/o llamadas grupales. La plataforma Arc Gis on líne figura como un soporte técnico y como herramienta que favorece el diseño de materiales y cumplir con los objetivos de aprendizaje y construir los productos finales. Para asegurar el acceso al curso y a la información los alumnos fueron invitados a través del correo electrónico institucional y les fue presentado el entorno virtual de aprendizaje, las acciones y aplicaciones colaborativas del curso. Se les mostro la estrategia didáctica, los objetivos de la materia, los contenidos y los accesos a las plataformas que les permite acceder de acuerdo al cronograma y planeación de las sesiones según sea el caso, asegurando su visibilidad y uso. Para la evaluación y el seguimiento al alumno se ha diseñado para que sea un proceso continuo y lineal, cuyo objetivo es valorar los logros en la adquisición del aprendizaje, procurando como lo menciona Edmiston y Borgman (2010) enfocar la evaluación fomentando la auto reflexión. Por lo que al inicio del curso los alumnos conocen las herramientas de evaluación como los Test de repaso que se presentan a lo largo del curso asincrónicos los foros de participación, como las listas de cotejo para entrega de productos y la rubrica evaluativa final, estas herramientas permiten ir más allá del cumplimento y entrega de un trabajo sino se busca trascender el 
procesamiento cognitivo centrándonos en como construye el alumno el conocimiento y la reflexión en torno a los instrumentos de evaluación para fortalecer la participación.

Por lo que en el diseño del curso a distancia a través de las TIC de Ordenamiento Territorial se buscó asegurar los diferentes criterios de la funcionalidad en lo referente a la interoperabilidad para interactuar con otras aplicaciones o programas, la seguridad como mecanismo para proteger la información dando solo acceso a las personas inscritas Institucionalmente y a controlando el acceso a los invitados, la escalabilidad para poder ir modificando las funcionalidades de acuerdo a las necesidades del curso y la disponibilidad de acuerdo a las necesidades para que algunos de los contenidos y acceso a información pueda ser descargable y trabajar sin red y por último importante desarrollar el trabajo colaborativo con mecanismos disponibles para propiciar la interacción entre los participantes, los canales abiertos para este propósito, los foros de discusión sincrónico, el chat permanente y el WhatsApp.

Se ha tratado que cada una de las herramientas elegidas puedan ser accesibles a todos los alumnos en cualquier dispositivo asegurando la portabilidad para que la herramienta principal de comunicación sea ejecutada en diferentes sistemas operativos asegurando adaptabilidad, sabiendo que la enseñanza de contenidos del área de conocimiento del ordenamiento territorial requiere de herramientas técnicas en el campo de la georreferenciación es que se puso a disposición del alumno el Arc Gis on line para codificar, y monitorear ensamblajes de datos y métodos provenientes de diferentes áreas del conocimiento información que atañe al territorio como estructura geográfica-espacial, y que sostenida por la combinación de la plataforma de Mapa digital de INEGI y de los contenidos que ofrece el Observatorio urbano Local de San Luis Potosí, como ente multidisciplinario y de investigación de la Facultad del Hábitat y su laboratorio satelital aseguran la enseñanza aprendizaje.

En lo que se refiere a la usabilidad cada una de las plataformas elegidas tiene su tutorial para mejorar la eficacia y la satisfacción de los usuarios. 


\section{La experiencia}

La materia de ordenamiento territorial en la carrera de diseño urbano y del paisaje exige desarrollar competencias profesionales en los alumnos en cuanto a la preparación de los procesos teórico-metodológicos para la elaboración de programas de ordenamiento territorial para crear cuadros competentes y que se ajusten a los que exige la ley de OT. Por lo que el objetivo general comprende Analizar los antecedentes y evolución histórica del concepto de Ordenamiento del Territorio, y sus diversas aplicaciones en el ámbito global, nacional, regional, urbano y municipal. Comprender las estrategias del Ordenamiento del Territorio, que incluyen la planeación urbana y regional, y planeación del desarrollo urbano municipal y manejar las herramientas conceptuales, metodológicas, técnicas y jurídicas del Ordenamiento del Territorio.

Ante el panorama Nacional de ordenamiento territorial los instrumentos de planeación integral proporcionan información estratégica para identificar potencialidades y conflictos para el uso del territorio, reconocer los peligros naturales y apoyar en la construcción de escenarios y modelo de ocupación territorial para así tomar decisiones para asegurar un desarrollo sustentable. Con esta perspectiva aunada a las disposiciones que deben ajustarse a la Ley General de asentamientos humanos, ordenamiento territorial y desarrollo urbano es que se concuerda la estrategia didáctica para que el alumno desarrolle las competencias para razonar a través del establecimiento de relaciones coherentes y sistematizables entre la información derivada de la experiencia y los marcos conceptuales y modelos explicativos derivados de los campos científicos y tecnológicos propios de la profesión, Implementando estrategias o procedimientos para llegar a un determinado resultado, asumiendo las propias responsabilidades bajo criterios de calidad y pertinencia hacia la sociedad.

La estructura de este curso está determinada por tres Unidades de Aprendizaje establecidas por el Plan de Estudios que corresponden a 17 semanas de tiempo por ciclo escolar, cada Unidad tiene un alcance intermedio de logro y desempeño para lograr desarrollar las competencias que implican al profesor como mediador entre el que aprende y el conocimiento,

Volumen 2. Número 2. Julio - Diciembre 2022 
por lo que los contenidos deben ir paulatinamente articulados a los saberes; el ser, el convivir, el hacer y el saber conocer, factores como la retroalimentación y la evaluación permanente ante un portafolio de evidencias que deben tener significancia para el alumno. Por lo que a lo largo del curso en la modalidad virtual debe considerarse la acción, el contenido y la condición identificando las herramientas TIC adecuadas para tal fin.

Imagen 4. Estructura del curso, Unidades de Aprendizaje y materiales de clase en MS Teams del curso ordenamiento territorial Facultad del Hábitat.

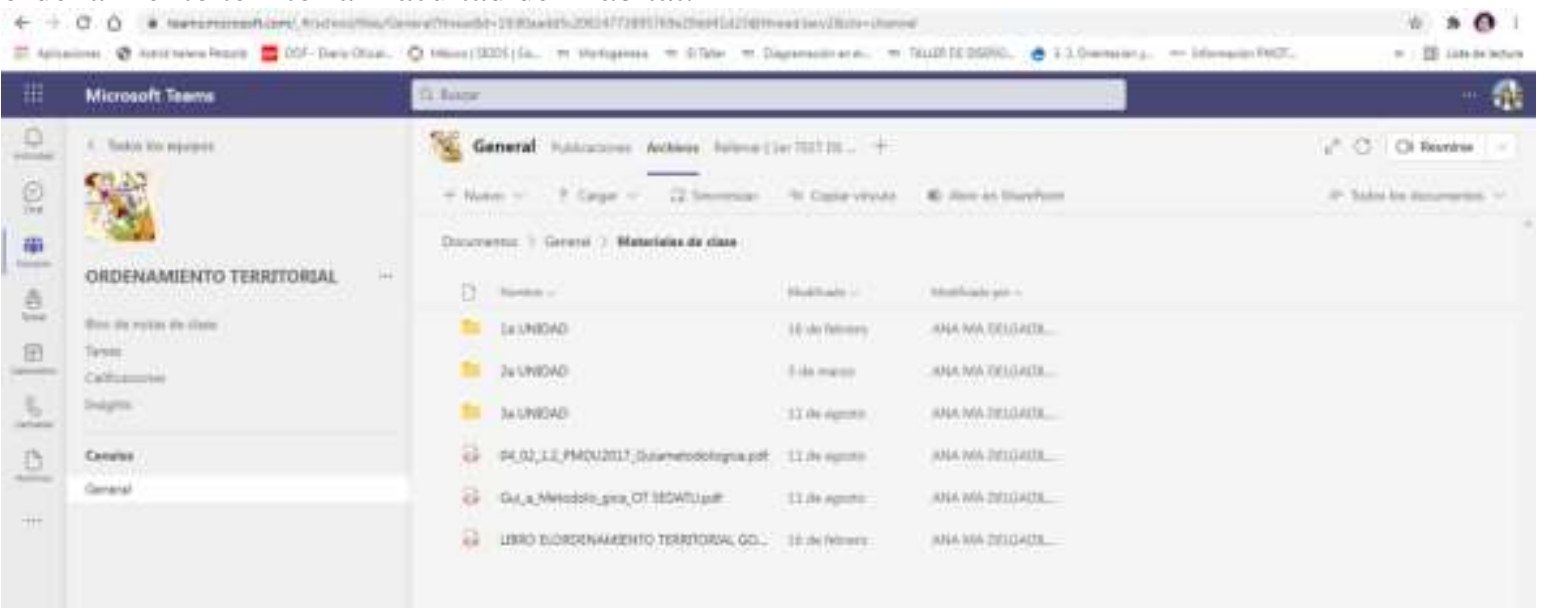

Imagen 5. Sesión Ms Teams del curso Ordenamiento territorial desplegada con los recursos de comunicación video compartiendo pantalla y chat permanente.

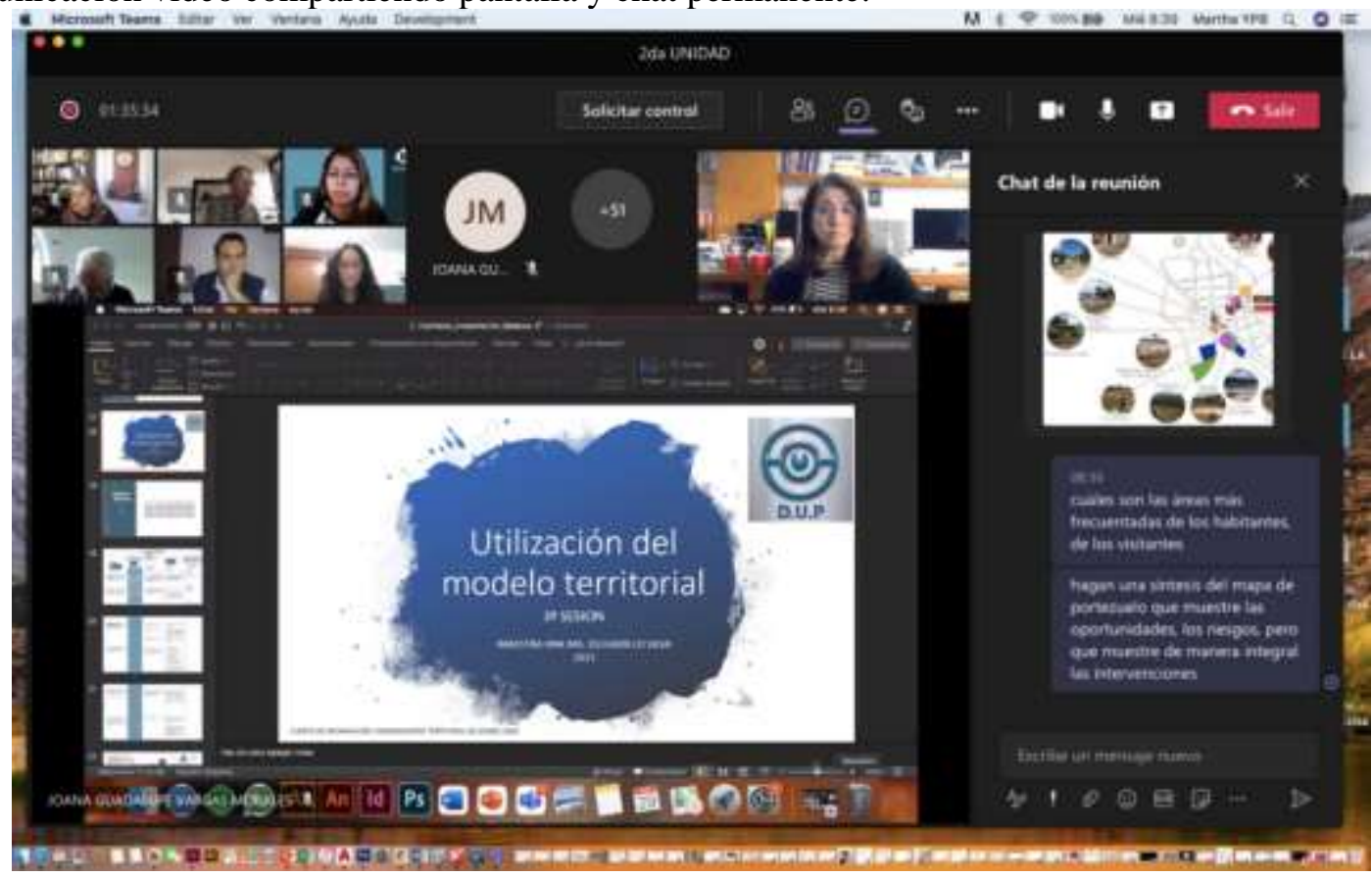


Fig. 2. Flujo del uso de las diversas plataformas propuestas para el curso de Ordenamiento territorial, Facultad del Hábitat, elaboración propia, 2021.

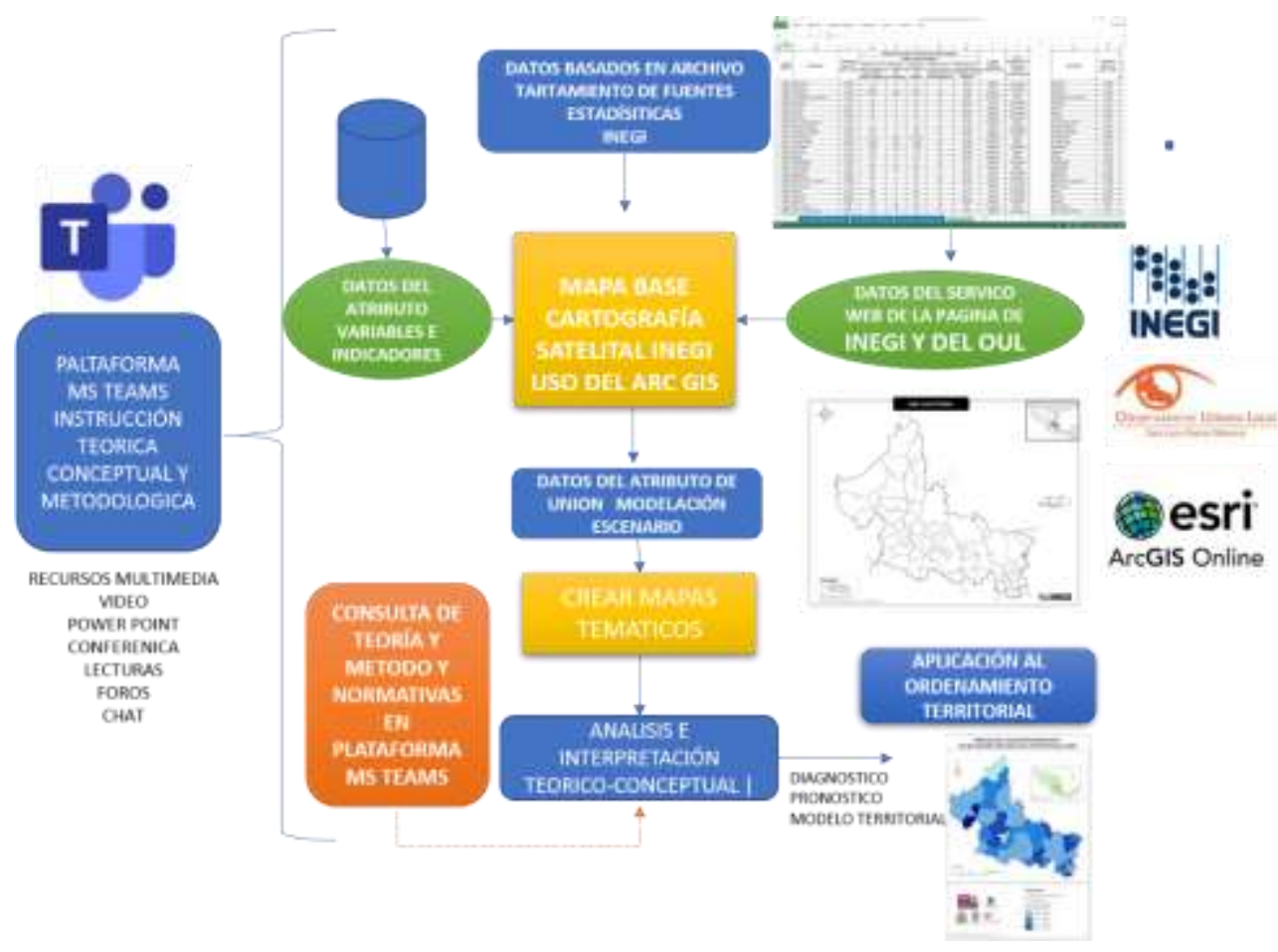

2.1 Por lo que la primera Unidad de aprendizaje aborda el marco de referencia conceptual del OT, los objetivos que se persiguen y las consideraciones teóricas a tomar en cuenta para la elaboración del POT.

\section{Estrategia didáctica de la primera Unidad}

Exposición, presentar la información y contenidos del curso. Presentar una conferencia de tipo informativo. Exponer resultados o conclusiones de una actividad.

\section{Técnica de aprendizaje}

Presentación de temas, foro, preguntas y respuestas, síntesis. Con base en preguntas llevar a los alumnos a la discusión y análisis de información pertinente a la materia. 


\section{Actividades}

Iniciar la discusión de un tema. Guiar la discusión del curso. Promover la participación de los alumnos. Generar controversia creativa en el grupo.

\section{Recursos TIC}

. Plataforma MS Teams: Instrucción didáctica, Archivos en materiales de clase: Foro, Video, presentaciones, lecturas y test de repaso; Chat permanente como foro de discusión.

Fig. 3. Objetivo, Estrategia didáctica y recursos TIC de la Primera Unidad de aprendizaje.

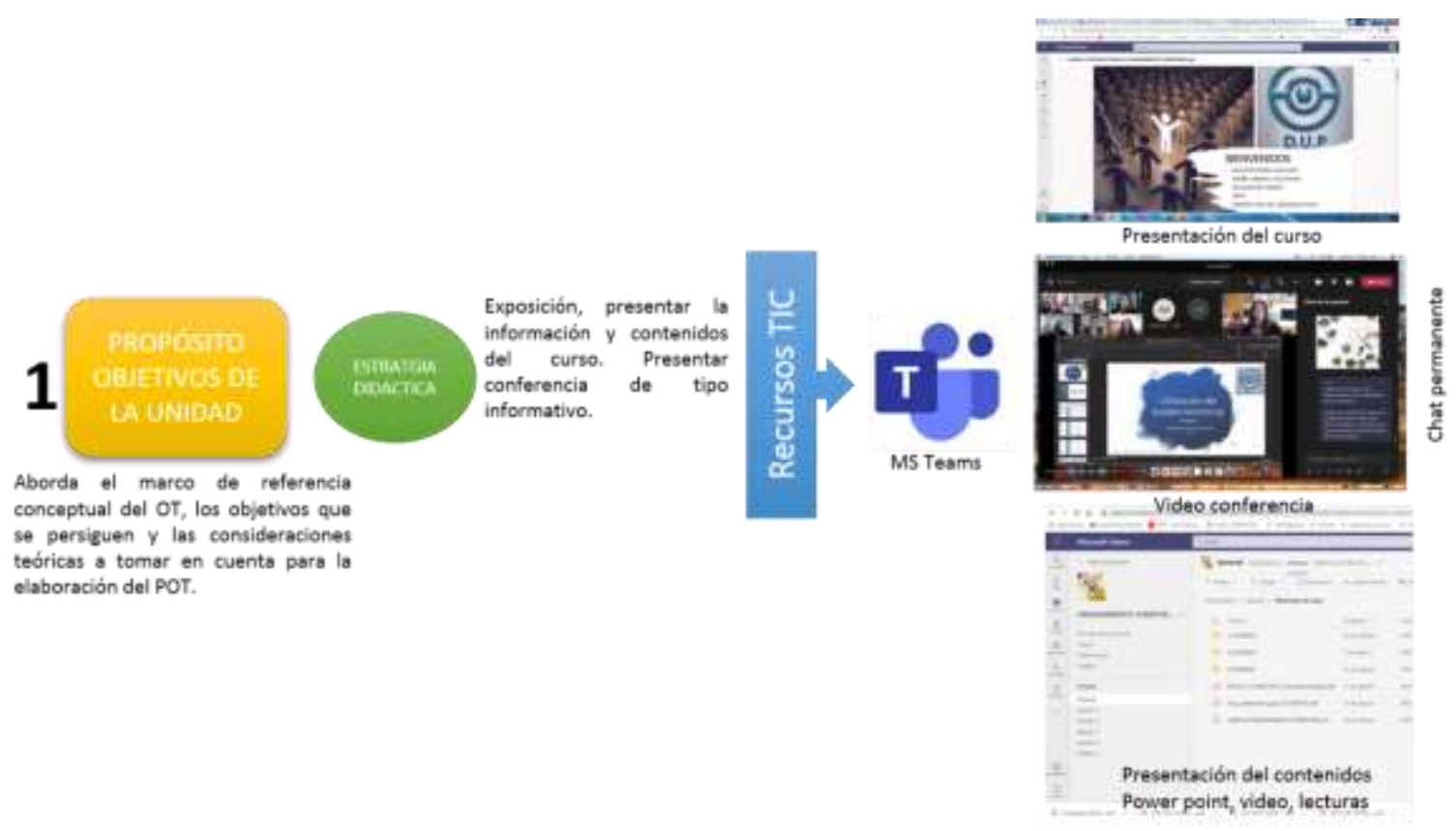

Elaboración de las autoras, curso Ordenamiento territorial 2021.

2.2 La segunda Unidad corresponde a identificar diseñar el programa de ordenamiento territorial, donde se aprende como se desarrolla el esquema general y las fases de las que se compone el POT, ejecución, fuentes de información y metodología a seguir. 


\section{Estrategia didáctica de la segunda unidad}

Enseñanza problemática; situaciones problemáticas territoriales, participación activa e independiente

\section{Técnica de aprendizaje}

Análisis de casos acerca de la realidad concreta del ambiente territorial

\section{Actividades}

Atender la instrucción didáctica del profesor, revisión de los conceptos para valorar los casos planteados, investigar y recopilar información necesaria desde la comprensión de las metodologías de OT, así como de las herramientas de aplicación mostradas en las conferencias con lo diversos Agentes expertos y tutoriales de las plataformas INEGI y Arc Gis online. Atender el foro de consulta.

\section{Recursos TIC}

. Plataforma MS Teams: Instrucción didáctica, Chat permanente como foro de discusión;

. Red social Whatsapp: comunicación constante;

- Plataforma OUL: Conocimiento de los alcances y objetivos del Observatorio en la Investigación documental y cartográfica del territorio

. Plataforma INEGI: Tutorial para el uso de la información estadística de datos, Conocimiento de los indicadores SCINSE 2020 Tutorial sobre Mapa digital y bases cartográficas para el análisis territorial.

- Plataforma ZOOM, Conferencia por Agentes de SEDUVOP "Marco normativo para la elaboración del POT, convenio de colaboración con OUL"

. Plataforma ARC GIS On line, Inscripción Institucional y tutorial 
Fig. 4 Objetivo, Estrategia didáctica y recursos TIC de la Segunda Unidad de aprendizaje.

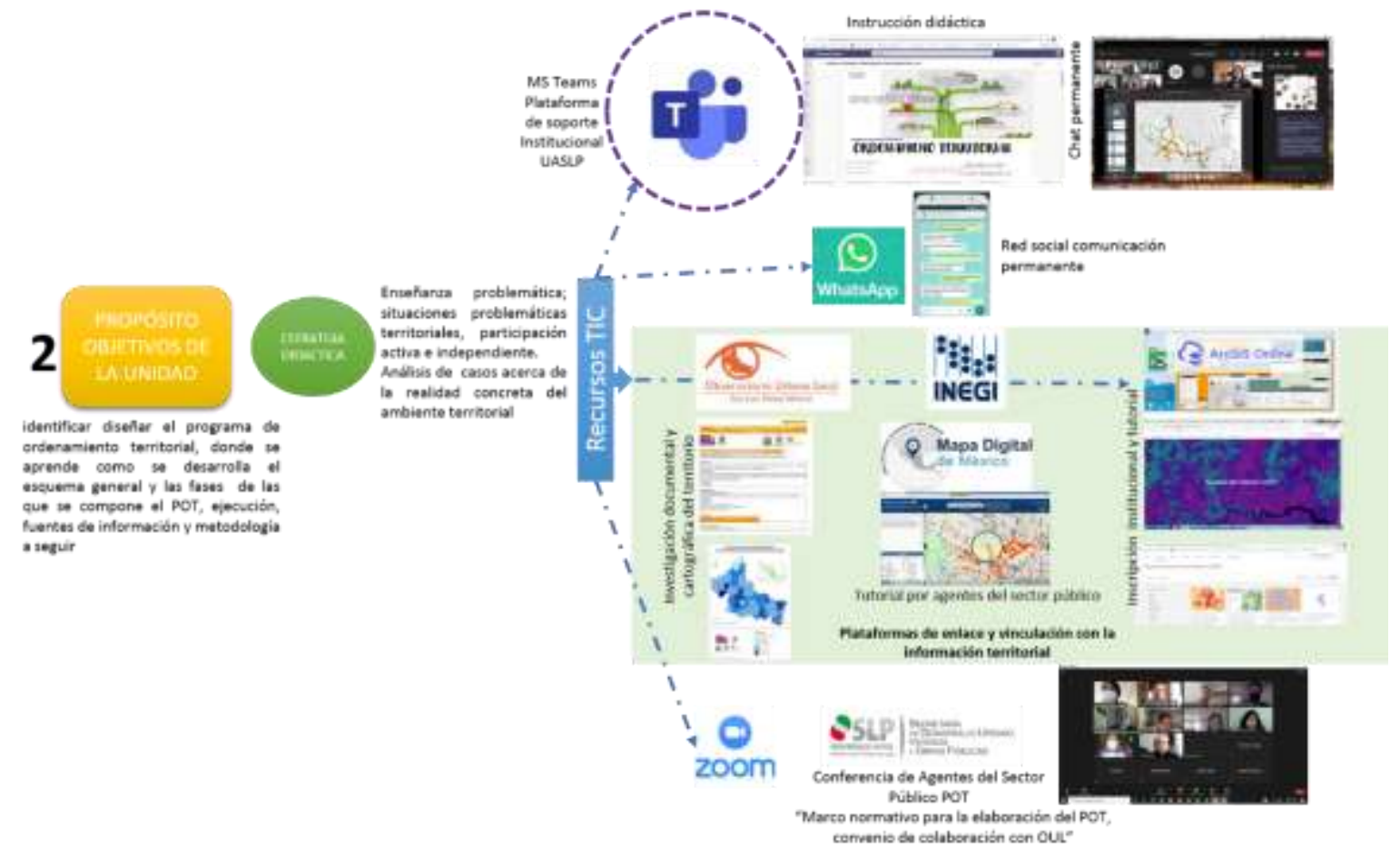

Elaboración de las autoras, curso Ordenamiento territorial 2021.

2.3 La tercera Unidad comprende la parte práctica de manera específica para el desarrollo de las cinco fases que comprende el desarrollo el POT, el alumno reconocerá como se constituye el territorio, sus etapas y procesos en la construcción territorial y que, a través de comprender las Estrategias Nacional, Regional y Locales de Planeación y Ordenamiento del Territorio e Identificar el Marco Jurídico del Ordenamiento del Territorio. Aplicará la Metodología utilizando información para analizar, codificar, y monitorear ensamblajes de datos y métodos provenientes de diferentes áreas del conocimiento por medio de las herramientas tecnológicas de información en los que respecta al territorio como estructura geográfica-espacial, marco que implica aplicar las normas vigentes para evaluar y hacer gestión territorial.

\section{Estrategia didáctica}

Aprendizaje colaborativo en comunidad virtual 


\section{Técnica Aprendizaje}

Resolución de problemas Aplicando lo aprendido, reafirmando conocimientos por proyecto Planificación de la tarea, Investigación, Análisis y la síntesis, elaboración del producto, Presentación del producto

\section{Actividades}

Búsqueda de la información para el análisis y síntesis de las variables e identificación de los indicadores asignados por equipo, que integran los subsistemas de orden territorial. Identificar las fuentes de información generada y provista por las instancias oficiales federales, locales, académicas y de investigación como aquella que genere el trabajo de campo, Construcción de la base de datos de indicadores, graficar de los datos, construcción de los Mapa temático, Consulta del marco teórico conceptual para el análisis espacial y territorial, desarrollo por escrito de la argumentación para el diagnóstico, posibles capacidades de carga de las distintas actividades de uso de cada una de las subunidades territoriales Igualmente en esta fase se llevan a cabo las evaluaciones de las amenazas naturales, la determinación de las vulnerabilidades así como la evaluación de los riesgos.

\section{Recursos Tic}

- Plataforma MS Teams: Instrucción didáctica, apertura de canal por equipo, apertura de carpeta en archivos subir productos, Chat permanente como foro de discusión;

. Whatsapp: crear chat del equipo, planificación de tareas grupales, comunicación constante;

. Plataforma OUL: Identificar indicadores y cartografía base;

. Plataforma INEGI: Uso de la información estadística de datos,

. Conexión a ARC GIS on line: Para la construcción del sistema de información geográfica de las variables y construcción del Mapa temático. 
Fig. 6 Objetivo, Estrategia didáctica y recursos TIC de la Tercera Unidad de aprendizaje.

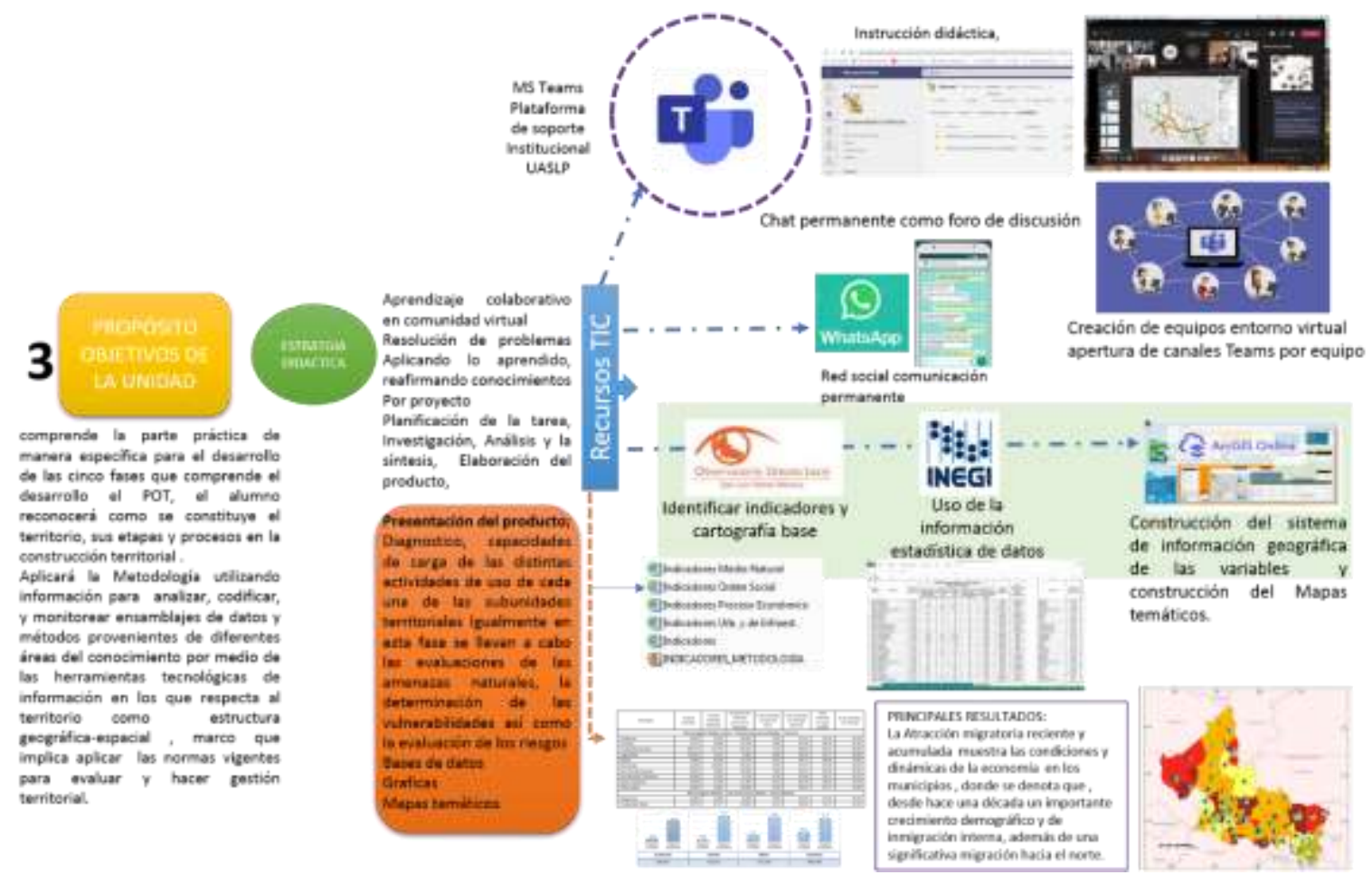

Elaboración de las autoras, curso Ordenamiento territorial 2021

\section{Análisis de resultados de la experiencia}

La manera en que se forman las nuevas generaciones depende del cambio de mentalidad y hasta de la cultura que seamos capaces de efectuar los profesores. La formación docente es esencial y con ello la necesidad de actualizarse permanentemente y perfeccionar los modos de actuación en nuevos entornos de aprendizaje, para ello el aprovechar las posibilidades que brindan las TIC recae en tarea indispensable.

En el mundo contemporáneo los conocimientos avanzan y se renuevan constantemente, es necesario pensar que los estudiantes no siempre son competentes para almacenar todos los conocimientos e información a la que están expuestos, sino habrá que prepararlos para que crearles competencias para buscar ese conocimiento y de aprender a aprender, para ello deben ampliar sus capacidades para adaptar nuevos procedimientos de aprendizaje. La integración 
de las TIC en el proceso de enseñanza aprendizaje para el ordenamiento territorial en los estudiantes de diseño urbano y del paisaje debe ser capaces de estar acorde a los nuevos entornos de aprendizaje, y en la mejora de la actuación en la práctica pedagógica. La integración de las TIC en el proceso de enseñanza aprendizaje se fundó como una necesidad sentida por toda la comunidad académica apoyados por la Institución universitaria para lograr nuevas metas en la calidad de las acciones de enseñanza y en las habilidades a formar en los estudiantes.

Podríamos decir que las ventajas que se encontraron en esta experiencia en el empleo de TIC en la formación de diseñadores urbanos y del paisaje para la elaboración de programas de ordenamiento territorial fue entre otras que el alumno fue capaz de tener una participación amplia y de forma colaborativa, lo cual lo vuelve más activo, optimizando el aprendizaje, ya que los contenidos y recursos en las plataformas están a su alcance a todo momento y el chat y los foros permanentes permitieron la constante comunicación, permitiendo a su vez mejorar en el campo de interés realizando muevas búsquedas investigando a través de la red.

La posibilidad de tener conferencias y tutoriales de diversos agentes expertos en la materia con acceso desde áreas remotas, contribuyendo a la eliminación de barreras espacio temporales, transformaron sustancialmente las formas y tiempos de la obtención de nueva información ante la experiencia de la interacción ya que puede tener lugar tanto de forma sincrónica como asincrónica, pues solo este hecho favoreció e incrementó los flujos de información y la colaboración más allá del aula física de la Universidad. Pues como hemos visto en esta experiencia la respuesta del alumno ante cualquier duda tuvo la posibilidad de tutorías individualizadas realizando consultas a través de los diferentes canales o enviar sus productos de tareas y proyectos en el tiempo definido y abierto para ello.

Esta nueva modalidad a distancia forzada al $100 \%$ por la contingencia sanitaria generó en principio un desconcierto a toda la comunidad pero como ya lo habíamos externado la UASLP venia ya haciendo un esfuerzo en la migración mixta por lo cual ya se habían desarrollado estrategias de capacitación a profesores, sin embargo al no tener el tiempo de reestructurar el

Volumen 2. Número 2. Julio - Diciembre 2022 
currículo los alumnos aunque motivados en un principio se vieron de pronto forzados a llevar la carga horaria de los cursos y horarios diseñados para la modalidad presencial por lo que existe una desventaja ya que se puede percibir que presentan un nivel elevado de estrés entre otras como las producidas por la falta de acceso a equipo adecuado o de fallas continuas de la red de internet.

\section{Conclusiones}

El uso de las Tecnologías de la información y la comunicación en la enseñanza distancia de diseñadores urbanos y del paisaje en la Facultad del Hábitat de la UASLP para la elaboración de programas de ordenamiento territorial se constituyó como una experiencia de interés para los estudiantes, generando expectativas positivas a través de proyectos reales posibilitando esta modalidad el acercamiento a información y a agentes del ámbito profesional, misma que fue posible por el uso de las TIC. La educación debe hacer frente a los retos que suponen las nuevas oportunidades que abren las tecnologías, que mejoran la manera de producir, organizar, difundir, controlar el saber y acceder al conocimiento. Debe garantizarse un acceso equitativo a estas tecnologías en todos los niveles de los sistemas de enseñanza, por lo que es necesario implementar estrategias de apoyo a los alumnos por parte de las Instituciones educativas. La experiencia de la migración a nuevos entornos de aprendizaje virtual y el uso de las TIC en los procesos de enseñanza aprendizaje anuncia la progresiva desaparición de las restricciones de espacio y de tiempo y la adopción de un modelo centrado en el estudiante. Las posibilidades que ofrecen las TIC permiten al docente ser copartícipe de la creación de entornos formativos en los cuales es notable la interacción multidireccional entre los participantes, aumentando así la construcción de los aprendizajes.

Las funciones de las TIC desde la perspectiva de los estudiantes presentan algunas ventajas pues le propicia interés, motivación entre los grupos de trabajo, la capacidad de abrirse a la discusión apoyados por herramientas comunicativas como el chat permanente, los foros de discusión o la red social del whatsapp utilizada con fines en este caso como medio de intercomunicación directa, desarrolla capacidades de iniciativa y mayor comunicación entre 
profesores y alumnos. Bricall (2000) y Márquez (2002) en Castro, S; Guzmán; Casado D. (2007).

Es preciso siempre estar atentos a las políticas de sociabilidad que están construidas a través de normas y reglas que permiten la adecuada comunicación manejada con propiedad y basada en respeto con el fin de inducir a un ambiente de aprendizaje tecnológico eficaz, cómodo y motivante, En estos ambientes el aprendizaje es activo, responsable, constructivo, intencional, complejo, contextual, participativo, interactivo y reflexivo (Kustcher y St. Pierre, 2001).

Frente al reto de lograr un impacto significativo, en la enseñanza asistida con TIC, podríamos especular debiera darse un giro total a las estrategias didácticas que hemos utilizado tradicionalmente para causar la adecuada apropiación de los aprendizajes. Resulta evidente a través de esta experiencia, que las estrategias utilizadas que hemos utilizado los docentes en la práctica presencial pueden ser de alguna manera fácil de adaptarlas a las herramientas y recursos que ofrecen los distintos sistemas. Para los que el profesor debe asumir un rol importante en la plataforma virtual para convertirse verdaderamente en un facilitador y lograr un importante impacto en la educación con el uso de TIC lo que plantea retos importantes que, no deben pasarse por alto.

Retos que se han encontrado en el entorno virtual, aunque se ha considerado que haya siempre interacción pueden ser la disminución de los aspectos afectivos, la mediación formativa, la socialización con esta nueva modalidad de aprendizaje, el desarrollo del trabajo en equipo, entre otros, y una apertura por parte de los profesores a aprender del nuevo entorno, donde muchas veces los estudiantes tienen más experiencia. El reto es un compromiso permanente por parte de profesores y alumnos apoyados institucionalmente Mantener y mejorar la calidad de la enseñanza en los entornos virtuales es un aspecto que no podemos descuidar, por lo tanto, no se puede pretender únicamente trasladar a la plataforma virtual los materiales y actividades que se utilizaban en el aula presencial, sino que debemos desarrollar y diseñar los apoyos multimedia que integren audio, imagen, texto $\mathrm{y}$, si es posible, que sean interactivas, así como actividades que promuevan el aprendizaje colaborativo mediante herramientas 
lúdicas, trabajos cuya finalidad sea el desarrollo del pensamiento crítico y el debate, debido a que es necesario atraer y motivar continuamente al estudiante a este entorno, darle las herramientas y guías necesarias para que desarrolle las actividades y alcance exitosamente las metas propuestas.

Es importante aclarar que las estrategias didácticas por sí solas no generan conocimiento y la plataforma virtual por sí sola no crea un espacio atractivo de aprendizaje, lo que hace la diferencia es la presencia del profesor como un facilitador que medie las unidades de aprendizaje y las temáticas del curso con estrategias didácticas creativas y que use, eficientemente, las herramientas que ofrece la plataforma. De esta forma, el docente generará un verdadero cambio en el aprendizaje apoyado en entornos virtuales. Para finalizar otro aspecto importante es que el éxito de una actividad en un entorno virtual depende en gran parte de los participantes, por lo tanto, no hay una estrategia didáctica $100 \%$ infalible, sino que se deben valorar las características del grupo y así decidir cuál es la que mejor responda a nuestras necesidades.

Los resultados de la experiencia solo nos compromete a mejorar para elegir las herramientas y estrategias didácticas que mantengan el interés y motivación y asegurar el aprendizaje sin deterioro del tiempo y el uso continuo que pudieran generar estrés al alumno, así mismo la vinculación con agentes externos expertos en el área nos deja una experiencia satisfactoria pues a través de su exposición e información al alcance de los alumnos generaron una aportación sobre los temas de territorio que contribuyen a la profesionalización de los alumnos en cuanto al ordenamiento territorial. 


\section{Referencias}

Asociación Nacional de Universidades e Instituciones de Educación Superior (ANUIES) (2001). Plan maestro de educación superior abierta y a distancia. Líneas estratégicas 15 para su desarrollo. México: ANUIES. http://es.scribd.com/doc/38786985/ANUIES-Plan-Maestrode-Educacion-SuperiorAbierta\#scribd

Castro, S; Guzmán; Casado D. (2007); Las Tic en los procesos de enseñanza y aprendizaje. Laurus, vol. 13, núm. 23, 2007, pp.213-234 Universidad Pedagógica Experimental Libertador Caracas, Venezuela. https://www.redalyc.org/pdf/761/76102311.pdf

Chávez, F. y Martínez, S. (2006, agosto). Evaluación educativa en las modalidades a distancia. Apertura, Revista de Innovación Educativa, vol. 6, núm. 4, pp.44-55. http://www.redalyc.org/articulo.oa?id=68800405

Delgado M.; Solano A.; (2009) Estrategias didácticas creativas en entornos virtuales para el aprendizaje, Revista Electrónica, Actualidades Investigativas en Educación, vol. 9, núm. 2, mayo-agosto, 2009, pp.1-21. http://www.redalyc.org/articulo.oa?id=44713058027

Dirección General de Materiales Educativos. Los Materiales Educativos en las Escuelas de Educación Básica. 2018, de Secretaría de Educación Pública. https://educacionbasica.sep.gob.mx/multimedia/RSC/BASICA/Documento/201709 /201709RSC-KHhL3KA6pm-PoliticasdematerialesBAJA.PDF

Edmiston, \& Borgman, 2010, "Using dramatic Inquir as literacy tool: Imaginative exploration in actual past, lived, and B. Edmiston. Pp.153. Ed. Literacy tools in 21s century classroom: Unleashing student agency. New York: Teachers college press. En Lapp, D., Moss B., (2012) Exeplary instruction in the middle grades: Teaching that. Pp.170 
Fullan, M.; smith, G. (1999). Technology and the Problem of Change. En Salinas, J. (2004) Innovación docente y uso de las TIC en la enseñanza universitaria pp.7; RUSC. Universities and Knowledge Society Journal. http://www.redalyc.org/articulo.oa?id=78011256001

Guevara, B. Guzmán J.C., Zambrano N. (2007) Comunidades Virtuales centradas en Usabilidad y Sociabilidad. https://www.researchgate.net/publication/228845714

Guevara B., Guzmán J. C., y Zambrano N., (2007), Comunidades Virtuales centradas en Usabilidad y Sociabilidad, Conferencia, primer encuentro Venezolano sobre Tecnologías de Información e Ingeniería de Software.

https://www.researchgate.net/publication/228845714_Comunidades_Virtuales_centradas_en _Usabilidad_y_Sociabilidad

Guía metodológica OT SEDATU, (2018). Secretaria de desarrollo agrario y territorial urbano, Gobierno Federal, México.

Kustcher N., y St. Pierre A., (2001) Pedagogía e Internet Aprovechamiento de las Nuevas Tecnologías. Editorial Trillas México DF. en Castro, S; Guzmán; Casado D. (2007); Las Tic en los procesos de enseñanza y aprendizaje. Laurus, vol. 13, núm. 23, 2007

Monsalve E., Valderrama A., (2019) Criterios para valorar herramientas TIC para la creación de Recursos Educativos Digitales, Programa Integración de Tecnologías a la Docencia Universidad de Antioquia. http://aprendeenlinea.udea.edu.co//ms/investigacion/mod/book/tool/print/index.php?id=1043 $7 \# \operatorname{ch} 249$

Powers, M. 1997. How to program a virtual community. Ziff-Davis Press. New York.

Rivera L., Rivera J., Reducindo I., Olvera M.A., (2016) Desarrollo de cursos de educación a distancia: una experiencia entre la UASLP y el INEGI, Revista Apertura, vol. 8, núm. 1, 
pp.1-17, 2016 Universidad de Guadalajara. Salinas, J., Pérez, A. y De Benito, B (2008).

Metodologías Centradas en el alumno para el aprendizaje en Red. España. Síntesis.

Salinas Ibáñez, J. (2008) Evolución de la tecnología y procesos de cambio e innovación educativa. Innovación educativa y uso de las TIC. Sevilla: Universidad Internacional de Andalucía, pp.127

Silvio, José "Las Comunidades Virtuales como conductoras de Aprendizaje Permanente". Instituto Internacional de la UNESCO para la Educación Superior en América Latina y el Caribe (IESALC) Venezuela. En: Rheingold, H. 1993. The virtual community. AddisonWesley. Reading, USA.

Pérez, García, E., (2014) Trabajo docente enriquecido mediante TIC, Las TIC al servicio de la formación universitaria integral, Secretaría Académica de la UASLP

Torres P.A., (2017) Saberes asociados al territorio a través de las TIC, Tesis en psicología, Universidad externado de Colombia facultad de ciencias Sociales y humanas. Recuperado https://bdigital.uexternado.edu.co/bitstream/handle/001/1031/DEA-spa-2017

Wong P.; (2009) Ordenamiento ecológico y ordenamiento territorial: retos para la gestión del desarrollo regional sustentable en el siglo XXI, RES Earta: Layout 1 10/22/2009 Page 11; Centro de Investigación en Alimentación y Desarrollo, A. C.pwong@ciad.mx 